\title{
A Note on Generalized Approximation Property
}

\author{
Antara Bhar and Manjul Gupta \\ Department of Mathematics and Statistics, Indian Institute of Technology, Kanpur 208016, India \\ Correspondence should be addressed to Antara Bhar; antarab@iitk.ac.in
}

Received 31 May 2013; Accepted 27 June 2013

Academic Editor: T. S. S. R. K. Rao

Copyright (C) 2013 A. Bhar and M. Gupta. This is an open access article distributed under the Creative Commons Attribution License, which permits unrestricted use, distribution, and reproduction in any medium, provided the original work is properly cited.

\begin{abstract}
We introduce a notion of generalized approximation property, which we refer to as $L_{s}$ - $\lambda$-AP possessed by a Banach space $X$, corresponding to an arbitrary Banach sequence space $\lambda$ and a convex subset $L_{s}(X)$ of $\mathscr{L}(X)$, the class of bounded linear operators on $X$. This property includes approximation property studied by Grothendieck, $p$-approximation property considered by Sinha and Karn and Delgado et al., and also approximation property studied by Lissitsin et al. We characterize a Banach space having $L_{s}-\lambda$-AP with the help of $\lambda$-compact operators, $\lambda$-nuclear operators, and quasi- $\lambda$-nuclear operators. A particular case for $\lambda=l_{p}(1 \leq p \leq \infty)$ has also been characterized.
\end{abstract}

\section{Introduction}

It is well known that the identity on an infinite dimensional Banach space is never compact, though it may be approximated by finite rank operators in the pointwise convergence topology, for instance, in the case when $X$ has a Schauder base. If the identity on $X$ is approximated uniformly on compact sets by finite rank operators, it leads to the notion of approximation property of $X$, studied systematically by Grothendieck [1] in 1955. Now there are many reformulations of this property, all of them involve either subspaces or ideals of operators, for example, class of finite rank operators, compact operators, and so forth. One may refer to [2-5] and references given therein.

In our recent work, using the duality theory of sequence spaces, we considered the notion of $\lambda$-compact sets corresponding to a suitably restricted sequence space $\lambda$ and studied $\lambda$-compact operators, specially their relationships with $\lambda$ summing, $\lambda$-nuclear, and quasi- $\lambda$-nuclear which were earlier considered by Ramanujan [6] in 1970. Replacing compact sets or $p$-compact sets by $\lambda$-compact sets, where $\lambda$ is a Banach sequence space and the class of finite dimensional operators or compact operators, and so forth, by an arbitrary convex subset $L_{s}(X)$ of the class $\mathscr{L}(X)$ of bounded linear operators on a Banach space, we define and study $L_{s}-\lambda$-AP of a Banach space $X$ in this paper. After giving preliminaries in the next section, we characterize this property in Section 3 and study its particular case for $\lambda=l_{p}$ in Section 4 . This property includes, as particular cases, approximation property, $p$ approximation property, compact approximation property, and subspace approximation property (cf. [2-5]).

\section{Preliminaries}

Throughout this paper, we denote by $\left(X,\|\cdot\|_{X}\right)$ a Banach space equipped with the norm $\|\cdot\|_{X}$ and by $X^{*}$ its topological dual equipped with the dual operator norm topology $\|\cdot\|_{X^{*}}$.

Let us begin with the basics of the sequence space theory, for which our reference is [7]. Let $\omega$ denote the family of all real or complex sequences, which is a vector space with usual pointwise addition and scalar multiplication, and let $\phi$ be the span of $e^{n}$ s $(n \geq 1)$, where $e^{n}$ is the $n$th unit vector in $\omega$; that is, $e^{n}=\{0,0, \ldots, 1,0,0, \ldots\}$, where 1 is the $n$th coordinate of the sequence $e^{n}$. A sequence space $\lambda$ is a subspace of $\omega$ containing $\phi$. Members of $\lambda$ are denoted by the symbols $\bar{\alpha}, \bar{\beta}$, and so forth, where $\bar{\alpha}=\left\{\alpha_{1}, \alpha_{2}, \alpha_{3}, \ldots\right\}$ and $\bar{\beta}=\left\{\beta_{1}, \beta_{2}, \beta_{3}, \ldots\right\}$. The $n$th section of $\bar{\alpha}$ for $n \in \mathbb{N}$ is written as $\bar{\alpha}^{(n)}$ and is defined as $\bar{\alpha}^{(n)}=$ $\left\{\alpha_{1}, \alpha_{2}, \ldots, \alpha_{n}, 0,0, \ldots\right\} ;$ that is, $\bar{\alpha}^{(n)}=\sum_{i=1}^{n} \alpha_{i} e^{i}$.

A sequence space $\lambda$ is called (i) symmetric if $\overline{\alpha_{\sigma}}=\left\{\alpha_{\sigma(i)}\right\} \in$ $\lambda$ whenever $\bar{\alpha}=\left\{\alpha_{i}\right\} \in \lambda$ and $\sigma \in \Pi$, where $\Pi$ is collection of all permutations of the set of natural numbers $\mathbb{N}$, (ii) monotone if $m_{0} \lambda \subseteq \lambda$, where $m_{0}=\operatorname{span}\{A\}$, and $A$ is the collection of all sequences consisting of zero and one, and (iii) 
normal or solid if $\bar{\beta}=\left\{\beta_{i}\right\} \in \lambda$ whenever $\left|\beta_{i}\right| \leq\left|\alpha_{i}\right|, i \geq 1$ for some $\bar{\alpha}=\left\{\alpha_{i}\right\} \in \lambda$.

The $\alpha$-dual, cross-dual, or Köthe-dual $\lambda^{\alpha}$ or $\lambda^{\times}$of $\lambda$ is defined as

$$
\begin{aligned}
\lambda^{\times} & \equiv \lambda^{\alpha} \\
& =\left\{\bar{\beta}=\left\{\beta_{i}\right\} \in \omega: \sum_{i \geq 1}\left|\alpha_{i} \beta_{i}\right| \text { converges, } \forall \bar{\alpha} \in \lambda\right\} .
\end{aligned}
$$

A sequence space $\lambda$ is said to be perfect if $\lambda=\lambda^{\times \times}=\left(\lambda^{\times}\right)^{\times}$. Every perfect sequence space is normal, and every normal sequence space is monotone.

A Banach sequence space $\left(\lambda,\|\cdot\|_{\lambda}\right)$ is called a $B K$-space provided that each of the projection maps $P_{i}: \lambda \rightarrow \mathbb{K}$, $P_{i}(\bar{\alpha})=\alpha_{i}$ is continuous, for $i \geq 1$, where $\mathbb{K}$ is the field of scalars and $\bar{\alpha}=\left\{\alpha_{1}, \alpha_{2}, \ldots\right\}$. A BK-space $\left(\lambda,\|\cdot\|_{\lambda}\right)$ is called an $A K$-space if $\bar{\alpha}^{(n)} \rightarrow \bar{\alpha}$, for each $\bar{\alpha} \in \lambda$.

For a BK-space $\left(\lambda,\|\cdot\|_{\lambda}\right)$, we define the dual norm on $\lambda^{\times}$ as follows:

$$
\|\bar{\beta}\|_{\lambda^{\times}}=\sup \left\{\sum_{i \geq 1}\left|\alpha_{i}\right|\left|\beta_{i}\right|: \bar{\alpha} \in \lambda,\|\bar{\alpha}\|_{\lambda} \leq 1\right\} .
$$

The space $\left(\lambda^{\times},\|\cdot\|_{\lambda^{\times}}\right)$can easily be shown to be a BK-space provided that $0<\sup _{n}\left\|e^{n}\right\|_{\lambda}<\infty$.

The norm $\|\cdot\|_{\lambda}$ is said to be (i) $k$-symmetric if $\|\bar{\alpha}\|_{\lambda}=$ $\left\|\bar{\alpha}_{\sigma}\right\|_{\lambda}$, for all $\sigma \in \Pi$ and (ii) monotone if $\|\bar{\alpha}\|_{\lambda} \leq\|\bar{\beta}\|_{\lambda}$ for $\bar{\alpha}, \bar{\beta}$ in $\lambda$ with $\left|\alpha_{i}\right| \leq\left|\beta_{i}\right|$, for all $i \geq 1$.

An Orlicz function $M$ is a continuous, convex function defined from $[0, \infty)$ to itself such that $M(0)=0, M(x)>0$ for $x>0$. Such function $M$ always has the integral representation

$$
M(x)=\int_{0}^{x} p(t) d t
$$

where $p(t)$, known as the kernel of $M$, is right continuous, nondecreasing function for $t>0$. Let us note that an Orlicz function $M$ is always increasing, and $M(x) \rightarrow \infty$ as $x \rightarrow$ $\infty$. Also $t p(t) \rightarrow \infty$ as $t \rightarrow \infty$, and $t p(t)=0$ for $t=0$ (cf. [4], page 139). However, $p(t)>0$ for $t=0$ is equivalent to the fact that the Orlicz sequence space $l_{M}$ (see the definition below) is isomorphic to $l_{1}$ (cf. [7], page 309). Therefore, we assume here that the kernel $p(t)$ has value 0 for $t=0$ and obviously $p(t) \rightarrow \infty$ as $t \rightarrow \infty$.

Given an Orlicz function $M$ with kernel $p$, define $q(s)=$ $\sup \{t: p(t) \leq s\}, s \geq 0$. Then $q$ possesses the same properties as $p$, and the function $N$ defined as $N(x)=\int_{0}^{x} q(t) d t$ is an Orlicz function. The functions $M$ and $N$ are called mutually complementary Orlicz functions. For such functions $M$ and $N$, we have Young's inequality: $x y \leq M(x)+N(y)$, for $x, y \geq 0$ and also $M(\alpha x) \leq \alpha M(x)$ for $0<\alpha<1$ (cf. [7]).

An Orlicz function $M$ is said to satisfy the $\Delta_{2}$-condition for small $x$ or at 0 , if, for each $k>1$, there exist $R_{k}>0$ and $x_{k}>0$ such that

$$
M(k x) \leq R_{k} M(x), \quad \forall x \in\left(0, x_{k}\right] .
$$

Corresponding to an Orlicz function $M$, the set $\tilde{l}_{M}$ is defined by

$$
\tilde{l}_{M}=\left\{\bar{\alpha} \in \omega: \sum_{i \geq 1} M\left(\left|\alpha_{i}\right|\right)<\infty\right\} .
$$

If $M$ and $N$ are mutually complementary functions, the Orlicz sequence space $l_{M}$ is defined as

$$
l_{M}=\left\{\bar{\alpha} \in \omega: \sum_{i \geq 1} \beta_{i} \alpha_{i} \text { converges, } \forall\left\{\beta_{i}\right\} \in \tilde{l}_{N}\right\} .
$$

An equivalent way of defining $l_{M}$ is

$$
l_{M}=\left\{\bar{\alpha} \in \omega: \sum_{i \geq 1} M\left(\frac{\left|\alpha_{i}\right|}{\rho}\right)<\infty \text { for some } \rho>0\right\} \text {. }
$$

Two equivalent norms on $l_{M}$ are given by

$$
\begin{aligned}
& \|\bar{\alpha}\|_{(M)}=\sup \left\{\left|\sum_{i \geq 1} \beta_{i} \alpha_{i}\right|: \sum_{i \geq 1} N\left(\left|\beta_{i}\right|\right) \leq 1\right\}, \\
& \|\bar{\alpha}\|_{M}=\inf \left\{\rho>0: \sum_{i \geq 1} M\left(\frac{\left|\alpha_{i}\right|}{\rho}\right) \leq 1\right\} ;
\end{aligned}
$$

indeed,

$$
\|\bar{\alpha}\|_{M} \leq\|\bar{\alpha}\|_{(M)} \leq 2\|\bar{\alpha}\|_{M}, \quad \text { for } \bar{\alpha} \in l_{M} .
$$

If $M$ and $N$ are mutually complementary Orlicz functions and $M$ satisfies $\Delta_{2}$-condition at 0 , then $\left(l_{M}\right)^{\times}=l_{N}$ (cf. [7]).

Corresponding to a sequence space $\lambda$ and a Banach space $X$ with its topological dual $X^{*}$ equipped with the operator norm topology generated by $\|\cdot\|$, the vector-valued sequence spaces $\lambda^{s}(X)$ and $\lambda^{w}(X)$ defined below, have been introduced and studied earlier in [6], under different notations. Indeed, we have

$$
\begin{gathered}
\lambda^{s}(X)=\left\{\bar{x}=\left\{x_{n}\right\} \subset X:\left\{\left\|x_{n}\right\|_{X}\right\} \in \lambda\right\}, \\
\lambda^{w}(X)=\left\{\bar{x}=\left\{x_{n}\right\} \subset X:\left\{f\left(x_{n}\right)\right\} \in \lambda, \forall f \in X^{*}\right\} .
\end{gathered}
$$

In case $\|\cdot\|_{\lambda}$ is a monotone norm, the space $\lambda^{s}(X)$ becomes a normed linear space with respect to the norm defined as

$$
\|\bar{x}\|_{\lambda}=\left\|\left\{\left\|x_{n}\right\|_{X}\right\}\right\|_{\lambda}, \quad \bar{x}=\left\{x_{n}\right\} \in \lambda^{s}(X) .
$$

However, for $\bar{x} \in \lambda^{w}(X)$, the norm on $\lambda^{w}(X)$ is defined as

$$
\begin{array}{r}
\|\bar{x}\|_{\lambda}^{w}=\sup \left\{\left\|\left\{f\left(x_{n}\right)\right\}\right\|_{\lambda}, f \in X^{*},\|f\| \leq 1\right\}, \\
\bar{x}=\left\{x_{n}\right\} \in \lambda^{w}(X),
\end{array}
$$

which can be proved to be finite by applying closed graph theorem. For the norm $\|\bar{y}\|_{\lambda^{\times}}^{w}$ on $\left(\lambda^{\times}\right)^{w}(X)$, we assume throughout that $0<\sup _{n}\left\|e^{n}\right\|_{\lambda}<\infty$ so that $\left(\lambda^{\times}\right)^{w}(X)$ equipped with this norm becomes a $K$-space.

Corresponding to a Banach sequence space $\lambda$ with $0<$ $\sup _{n}\left\|e^{n}\right\|_{\lambda}<\infty$ and a Banach space $X$, we have introduced the concept of $\lambda$-compact sets and $\lambda$-compact operators in 
our work [8]. A subset $K$ of $X$ is said to be $\lambda$-compact if there exists $\bar{x}=\left\{x_{n}\right\} \in \lambda^{s}(X)$ such that $K \subseteq \lambda-\operatorname{co}\left\{x_{n}\right\}=$ $\left\{\sum_{n \geq 1} \alpha_{n} x_{n}: \bar{\alpha}=\left\{\alpha_{n}\right\} \in B_{\lambda^{\times}}\right\}$. For $\lambda=l_{p}, 1 \leq p \leq \infty, \lambda$ compact sets are nothing but $p$-compact sets studied in [9]. [8].

Concerning the $\lambda$-compact sets, we have the following

Theorem 1. Let $\lambda$ be a normal sequence space equipped with a monotone norm $\|\cdot\|_{\lambda}$ satisfying the condition $0<\sup _{n}\left\|e^{n}\right\|_{\lambda}<$ $\infty$. Also let $\left(\lambda,\|\cdot\|_{\lambda}\right)$ be an $A K-B K$-reflexive sequence space. Then for $\bar{x}=\left\{x_{n}\right\} \in \lambda^{s}(X)$, the set $\left\{\sum_{n \geq 1} \alpha_{n} x_{n}: \bar{\alpha}=\left\{\alpha_{n}\right\} \in\right.$ $\left.B_{\lambda^{\times}}\right\}$is norm closed in $X$.

For Banach spaces $X$ and $Y$, the symbol $\mathscr{L}(X, Y)$ denotes the class of all bounded linear operators from $X$ to $Y$, and $\mathscr{L}$ denotes the collection of all bounded operators between any pair of Banach spaces. The notation $\mathfrak{F}(X, Y), K(X, Y)$, and $W(X, Y)$, respectively, stand for collection of all finite rank, compact, and weakly compact operators from $X$ to $Y$. For $Y=$ $X$, we write $\mathscr{L}(X, Y) \equiv \mathscr{L}(X), \mathfrak{F}(X, Y) \equiv \mathfrak{F}(X)$, and so forth.

The space $\mathscr{L}(X, Y)$ endowed with the locally convex topology $\tau_{p}(1 \leq p \leq \infty)$ has been studied in [10], where $\tau_{p}$ is the topology of uniform convergence on $p$-compact sets in $X$. Concerning the dual space $\left(\mathscr{L}(X, Y), \tau_{p}\right)^{*}$ for $1<p<\infty$, Choi and Kim [10] have proved the following.

Theorem 2. Let $1<p, q<\infty$, and $1 / p+1 / q=1$. Then $\left(\mathscr{L}(X, Y), \tau_{p}\right)^{*}$ consists of all linear functionals $\varphi$ of the form

$$
\varphi(T)=\sum_{j \geq 1} \sum_{n \geq 1} \alpha_{n}^{j} g_{j}\left(T x_{n}\right)
$$

where $\bar{x}=\left\{x_{n}\right\} \in l_{p}^{s}(X) ; \bar{\alpha}^{j}=\left\{\alpha_{n}^{j}\right\}_{n \geq 1}$ from $l_{q}$, for each $j \in \mathbb{N}$ and $\left\{g_{j}\right\} \subseteq Y^{*}$ with $\sum_{j \geq 1}\left\|\bar{\alpha}^{j}\right\|_{q}\left\|g_{j}\right\|<\infty$.

Let $\lambda$ be a normal BK-sequence space equipped with the norm $\|\cdot\|_{\lambda}$ satisfying $0<\inf _{n}\left\|e^{n}\right\|_{\lambda} \leq \sup _{n}\left\|e^{n}\right\|_{\lambda}<\infty$. An operator $T \in \mathscr{L}(X, Y)$ is said to be

(i) $\lambda$-compact operator if $T\left(B_{X}\right)$ is a $\lambda$-compact set in $Y$; that is, $T$ maps bounded sets in $X$ to $\lambda$-compact sets in $Y$,

(ii) $\lambda$-nuclear if $T$ has the representation

$$
T x=\sum_{n \geq 1} \alpha_{n}<x, f_{n}>y_{n}
$$

where $\left\{f_{n}\right\} \subseteq X^{*}$ with $\left\|f_{n}\right\|_{X^{*}} \leq 1$, for each $n$; $\bar{y}=$ $\left\{y_{n}\right\} \in\left(\lambda^{\times}\right)^{w}(Y)$ with $\|\bar{y}\|_{\lambda^{\times}}^{w} \leq 1$ and $\bar{\alpha}=\left\{\alpha_{n}\right\} \in \lambda$,

(iii) quasi- $\lambda$-nuclear if there exists $\left\{f_{n}\right\} \subseteq X^{*}$ such that $\bar{f}=$ $\left\{f_{n}\right\} \in \lambda^{s}\left(X^{*}\right)$ and $\|T x\| \leq\left\|\left\{f_{n}(x)\right\}\right\|_{\lambda}$, for each $x \in X$.

The symbols $K_{\lambda}(X, Y), N_{\lambda}(X, Y)$, and $Q N_{\lambda}(X, Y)$, respectively, denote the collection of all $\lambda$-compact, $\lambda$-nuclear, and quasi- $\lambda$-nuclear operators from $X$ to $Y$.

For the relationships of $\lambda$-compact, $\lambda$-nuclear, and quasi$\lambda$-nuclear operators, we have the following [8].
Theorem 3. Let $\left(\lambda,\|\cdot\|_{\lambda}\right)$ be a normal, symmetric, BKsequence space with $0<\inf _{n}\left\|e^{n}\right\|_{\lambda} \leq \sup _{n}\left\|e^{n}\right\|_{\lambda}<\infty$. Consider the following.

(i) For any pair of Banach spaces $X$ and $Y$, if $T \in \mathscr{L}(X, Y)$ is a $\lambda$-nuclear operator, then $T^{*} \in \mathscr{L}\left(Y^{*}, X^{*}\right)$ is a $\lambda$ compact operator;

(ii) if $T \in \mathscr{L}(X, Y)$ is a $\lambda$-compact operator, then $T^{*} \epsilon$ $\mathscr{L}\left(Y^{*}, X^{*}\right)$ is a quasi- $\lambda$-nuclear operator. [11].

For $\lambda=l_{p}(1 \leq p<\infty)$ and $T \in \mathscr{L}(X, Y)$, we quote from

Proposition 4. (i) $T \in K_{p}(X, Y)$ if and only if $T^{*} \in$ $Q N_{p}\left(Y^{*}, X^{*}\right) ; T \in Q N_{p}(X, Y)$ if and only if $T^{*} \in K_{p}\left(Y^{*}, X^{*}\right)$;

(ii) $T^{*} \in Q N_{p}\left(Y^{*}, X^{*}\right)$ if and only if there exists $\left\{y_{n}\right\} \in$ $l_{p}^{s}(Y)$ such that $\left\|T^{*} g\right\| \leq\left\|\left\{g\left(y_{n}\right)\right\}\right\|_{p}$, for all $g \in Y^{*}$.

We also make use of the following result from [6].

Theorem 5. Let $\lambda$ be a symmetric normal $A K-B K$-sequence space equipped with a monotone norm $\|\cdot\|_{\lambda}$ satisfying $0<$ $\sup _{n}\left\|e^{n}\right\|_{\lambda}<\infty$. Then $T \in Q N_{\lambda}(X, Y)$ implies that $T \in$ $N_{\lambda}(X, Y)$ if the range space $Y$ has extension property; that is, for every injection $J \in \mathscr{L}\left(X_{0}, X\right)$ and every operator $S_{0} \in$ $\mathscr{L}\left(X_{0}, Y\right)$, there exists an extension $S \in \mathscr{L}(X, Y)$ such that $\left\|S_{0}\right\|=\|S\|$ and $S_{0}=S J$.

For the salient features on operator ideals, one is referred to $[12]$.

Writing $\mathscr{A}(X, Y)=\mathscr{A} \cap \mathscr{L}(X, Y)$, a subset $\mathscr{A}$ of $\mathscr{L}$ is said to be an operator ideal if it satisfies the following conditions:

(i) $A$ contains all finite rank operators;

(ii) $T+S \in \mathscr{A}(X, Y)$ for $S, T \in \mathscr{A}(X, Y)$;

(iii) if $T \in \mathscr{A}(X, Y)$ and $S \in \mathscr{L}(Y, Z)$, then $S T \in \mathscr{A}(X, Z)$, and also if $T \in \mathscr{L}(X, Y)$ and $S \in \mathscr{A}(Y, Z)$, then $S T \in$ $\mathscr{A}(X, Z)$.

The collection $\mathscr{A}(X, Y)$, for a given pair of Banach spaces $X$ and $Y$, is called a component of $\mathscr{A}$.

It has been shown in $[6,8]$ that the collections of $\lambda$ compact, $\lambda$-nuclear, and quasi- $\lambda$-nuclear operators from $X$ to $Y$ are operator ideals for suitably chosen sequence space $\lambda$.

For an operator ideal $\mathscr{A}$, the dual operator ideal $\mathscr{A}^{d}$ is defined as the one of which the component $\mathscr{A}^{d}(X, Y)$ is given by

$$
\mathscr{A}^{d}(X, Y)=\left\{T \in \mathscr{L}: T^{*} \in \mathscr{A}\left(Y^{*}, X^{*}\right)\right\}
$$

For an operator ideal $\mathscr{A}$, the subspace $\mathscr{A}^{w^{*}}\left(X^{*}, Y\right)$ of the component $\mathscr{A}\left(X^{*}, Y\right)$ is defined as

$$
\begin{aligned}
\mathscr{A}^{w^{*}}\left(X^{*}, Y\right)=\{ & T \in \mathscr{A}\left(X^{*}, Y\right): \\
& \left.T \text { is } \sigma\left(X^{*}, X\right)-\sigma\left(Y, Y^{*}\right) \text { continuous }\right\} .
\end{aligned}
$$




\section{3. $L$-Subset $\lambda$-Approximation Property}

Throughout this section we denote by $X$, a Banach space and by $\lambda$, a BK-sequence space equipped with a norm $\|\cdot\|_{\lambda}$ such that $0<\sup _{n}\left\|e^{n}\right\|_{\lambda}<\infty$. Let $L_{s}(X)$ be a convex subset of $\mathscr{L}(X)$. Recalling the definition of $\lambda$-compact sets in $X$ from the previous section, we introduce the following.

Definition 6. A Banach space $X$ is said to have $L$-subset $\lambda$ approximation property $\left(L_{s}-\lambda\right.$-AP) if given $\varepsilon>0$ and any $\lambda$-compact set $K$ in $X$; there exists $R \in L_{s}(X)$ such that $\sup _{x \in K}\|R x-x\|_{X}<\varepsilon$; that is, the identity map on $X$ is approximated uniformly on a $\lambda$-compact set by a member of $L_{s}(X)$.

If $L_{s}(X)=\mathfrak{F}(X)$ and $\lambda=\mathcal{c}_{0}$, it is the approximation property studied by Grothendieck [1]. If $L_{s}(X)=\mathfrak{F}(X)$ and $\lambda=l_{p}$, it is the $p$-approximation property (cf. $[3,9]$ ), and if $L_{s}(X)=K(X)$ and $\lambda=c_{0}$, it is the compact approximation property (cf. [4]).

Theorem 7. Let $\left(\lambda,\|\cdot\|_{\lambda}\right)$ be an $A K-B K$-reflexive sequence space such that the norm $\|\cdot\|_{\lambda}$ is monotone satisfying the condition $0<\sup _{n}\left\|e^{n}\right\|_{\lambda}<\infty$. Assume that $\lambda$ is also monotone. Then for a convex subset $L_{s}(X)$ of $\mathscr{L}(X)$, the following conditions are equivalent.

(i) $X$ has $L_{s}-\lambda$-AP.

(ii) For any Banach space $Y$ and for any $T \in K_{\lambda}(Y, X)$, $T \in \overline{\left\{R T: R \in L_{s}(X)\right\}}{ }^{\|\cdot\|}$, the closure being considered with respect to the norm $\|\cdot\|$ in $\mathscr{L}(Y, X)$.

Proof. (i) $\Rightarrow$ (ii) Let $T \in K_{\lambda}(Y, X)$. Then $T\left(B_{Y}\right)$ is $\lambda$-compact set in $X$. Since $X$ has $L_{s}-\lambda$-AP, for $\varepsilon>0$ there exists $R_{0} \in$ $L_{s}(X)$ such that $\sup _{x \in T\left(B_{Y}\right)}\left\|x-R_{0} x\right\|<\varepsilon \Rightarrow\left\|T-R_{0} T\right\|<\varepsilon$. Thus $T \in \overline{\left\{R T: R \in L_{s}(X)\right\}}\|\cdot\|$.

(ii) $\Rightarrow$ (i) For proving (i), consider a $\lambda$-compact set $K$ of the form $K=\lambda-\operatorname{co}\left\{x_{n}\right\}$ for some $\bar{x}=\left\{x_{n}\right\} \in \lambda^{s}(X)$. In view of Theorem $1, K$ is norm closed and so it is complete. Hence the space $Y=\bigcup_{n \geq 1} n K$ is a Banach space with respect to the norm \|\|$\cdot \|$ defined as \|\|$y \|=\inf \{\rho>0: y \in \rho K\}$ for $y \in Y$. As $K$ is the unit ball of $Y$, the inclusion map $I$ from $Y$ to $X$ is $\lambda$-compact; that is, $I \in K_{\lambda}(Y, X)$. Therefore by hypothesis, given any $\varepsilon>0$, we can find that $R_{0} \in L_{s}(X)$ such that

$$
\left\|I-R_{0}\right\|=\sup _{x \in K}\left\|x-R_{0} x\right\|<\varepsilon .
$$

This proves that $X$ has $L_{s}-\lambda$-AP.

The above result leads to the following.

Proposition 8. Let $\left(\lambda,\|\cdot\|_{\lambda}\right)$ be a sequence space as in Theorem 7 , and let $L_{s}(X)$ be a convex subset of $\mathscr{L}(X)$. Then $X$ has $L_{s}-\lambda$-AP if $T \in{\overline{\left\{T R^{*}: R \in L_{s}(X)\right\}}}^{\|\cdot\|}$, for any Banach space $Y$ and any $T \in Q N_{\lambda}\left(X^{*}, Y\right)$.

Proof. Let $T \in K_{\lambda}(Y, X)$ where $Y$ is an arbitrary Banach space. Then $T^{*} \in Q N_{\lambda}\left(X^{*}, Y^{*}\right)$ by Theorem 3(ii). Hence for any $\varepsilon>0$, there exists $R_{0} \in L_{s}(X)$ such that $\| T^{*}-$
$T^{*} R_{0}^{*} \|<\varepsilon$, by hypothesis. Thus $\left\|T-R_{0} T\right\|<\varepsilon$; that is, $T \in{\overline{\left\{R T: R \in L_{s}(X)\right\}}}^{\|\cdot\|}$. Now apply Theorem 7 to get that $X$ has $L_{s}-\lambda$-AP.

If the Banach spaces $X$ and $Y$ are restricted further, we get the converse of Proposition 8 in the following form.

Proposition 9. Let $\left(\lambda,\|\cdot\|_{\lambda}\right)$ be as in Proposition 8. Assume that $X$ is a reflexive Banach space and $Y$ is a Banach space having extension property. If $X$ has $L_{s}-\lambda-A P$, then $T \in$ $\overline{\left\{T R^{*}: R \in L_{s}(X)\right\}}\|\cdot\|$, for any $T \in Q N_{\lambda}\left(X^{*}, Y\right)$.

Proof. Let $T \in Q N_{\lambda}\left(X^{*}, Y\right)$. Then $T \in N_{\lambda}\left(X^{*}, Y\right)$ by Theorem 5, and so $T^{*} \in K_{\lambda}\left(Y^{*}, X\right)$ by Theorem 3(i). By hypothesis and Theorem 7 , for $\varepsilon>0$, there exists $R_{0} \in L_{s}(X)$ such that $\left\|T-T R_{0}^{*}\right\|=\left\|T^{*}-R_{0} T^{*}\right\|<\varepsilon$; that is, $T \in$ ${\overline{\left\{T R^{*}: R \in L_{s}(X)\right\}}}^{\|\cdot\|}$.

For the dual $X^{*}$ of $X$, we introduce the following.

Definition 10. Let $X$ be a Banach space with its topological dual $X^{*}$ equipped with the operator norm topology, and let $L_{s}(X)$ be a convex subset of $\mathscr{L}(X)$. Then $X^{*}$ is said to have $L_{s^{-}}$ $\lambda$ approximation property with conjugate operator $\left(L_{s}^{*}-\lambda\right.$-AP) if, for any $\lambda$-compact set $K^{*}$ of $X^{*}$ and $\varepsilon>0$, there exists $R_{0} \in$ $L_{s}(X)$ such that $\sup _{f \in K^{*}}\left\|R_{0}^{*} f-f\right\|<\varepsilon$; that is, the identity operator on $X^{*}$ is approximation by a conjugate operator of a member in $L_{s}(X)$, uniformly on $\lambda$-compact subset of $X^{*}$.

Concerning $L_{s}^{*}-\lambda$-AP of $X^{*}$, we prove the following.

Theorem 11. Let $\left(\lambda,\|\cdot\|_{\lambda}\right)$ and $L_{s}(X)$ be as in Theorem 7 .

(i) If $Y$ is a Banach space with extension property and $X^{*}$ has $L_{s}^{*}-\lambda$-AP, then $T \in{\overline{\left\{T R: R \in L_{s}(X)\right\}}}^{\|\cdot\|}$ for any $T \in$ $Q N_{\lambda}(X, Y)$.

(ii) Let $X$ be a reflexive Banach space. If, for any Banach space $Y$ and $T \in Q N_{\lambda}(X, Y), T \in{\overline{\left\{T R: R \in L_{s}(X)\right.}}^{\|\cdot\|}$, then $X^{*}$ has $L_{s}^{*}-\lambda$-AP.

Proof. Omitted as these are analogous to the proofs of Propositions 8 and 9.

Remark 12. Let us note here that the restriction on $\lambda$ is such that we may include the Orlicz sequence spaces $l_{M}$, where $M$ is an Orlicz function satisfying $\Delta_{2}$-condition at zero. One may call such approximation property as $L_{s}-l_{M}$ AP and may be worth investigating further. However, for $L_{s}(X)=\mathfrak{F}(X)$, we illustrate $l_{M}$-AP by the following.

Example 13. We consider Orlicz functions $M_{1}(u)=$ $u^{2} /(\log (e+u))$ and $M_{2}(u)=u\left(e^{u}-1\right)$, for $u \geq 0$. Then $l_{M_{1}}=l_{2}=l_{M_{2}}$ (cf. [13]). Therefore $l_{N_{1}}=l_{2}=l_{N_{2}}$, where $N_{i}$ are the complementary Orlicz functions of $M_{i}$, respectively, for $i=1,2$. Let $K$ be an $l_{N_{1}}$-compact set in $X$. Then there exists $\bar{x}=\left\{x_{n}\right\} \in l_{N_{1}}^{s}(X)=l_{2}^{s}(X)$ such that $K \subseteq\left\{\sum_{n \geq 1} \alpha_{n} x_{n}\right.$ : $\left.\bar{\alpha}=\left\{\alpha_{n}\right\} \in B_{l_{M_{1}}}\right\}$. As $\|\bar{\alpha}\|_{M_{1}} \leq\|\bar{\alpha}\|_{2} \leq C\|\bar{\alpha}\|_{M_{1}}$, we have 
$K \subseteq\left\{\sum_{n \geq 1} \alpha_{n}\left(C x_{n}\right): \bar{\alpha}=\left\{\alpha_{n}\right\} \in B_{l_{2}}\right\}$. Thus every $l_{N_{1}}{ }^{-}$ compact set is $l_{2}$-compact set. Hence every Banach space has $l_{N_{1}}$-approximation property (cf. [9], page 27). Similarly we can show that every Banach space has $l_{N_{2}}$-approximation property.

\section{4. $L$-Subset $p$-Approximation Property}

In this section we study $L_{s}$ - $p$-approximation property which is a particular case of $L$-subset $\lambda$ approximation property for $\lambda=l_{p}(1 \leq p \leq \infty)$. Throughout this section, we denote by $L_{s}(X)$ a convex subset of $\mathscr{L}(X)$, where $X$ is a Banach space. Let us begin with the following.

Definition 14. Let $X$ be a Banach space with $X^{*}$ as its topological dual equipped with the operator norm topology. Then (i) $X$ is said to have $L_{s}(X)$-p-approximation property $\left(L_{s}\right.$-p-AP) if, for $\varepsilon>0$ and a $p$-compact subset $K$ of $X$, there exists $R_{0} \in L_{s}(X)$ such that $\sup _{x \in K}\left\|R_{0} x-x\right\|_{X}<\varepsilon$, and (ii) $X^{*}$ is said to have $L_{s}(X)$-p-approximation property with conjugate operator $\left(L_{s}^{*}-p\right.$-AP) if, for any $\varepsilon>0$ and a $p$-compact set $K^{*}$ in $X^{*}$, there exists $R_{0} \in L_{s}(X)$ such that $\sup _{f \in K^{*}}\left\|R_{0}^{*} f-f\right\|<\varepsilon$.

Characterizing Banach spaces having $L_{s}-p$-AP, we have the following.

Theorem 15. The following conditions are equivalent.

(i) $X$ has $L_{s}-p-A P$.

(ii) For any Banach space $Y$ and any $T \in K_{p}(Y, X), T \in$ ${\overline{\left\{R T: R \in L_{s}(X)\right\}}}^{\|\cdot\|}$.

(iii) For any Banach space $Y$ and any $T \in K_{p}(Y, X), T \in$ $\overline{\left\{R T: R \in L_{s}(X)\right\}^{\tau}}$, where $\tau$ is the topology of uniform convergence on compact subsets of $Y$.

Proof. (i) $\Leftrightarrow$ (ii) follows from Theorem 7 .

(ii) $\Rightarrow$ (iii) is obvious as norm topology is finer than $\tau$.

(iii) $\Rightarrow$ (i) The proof of this implication is almost the same as that of Theorem $1(c \Rightarrow a)$ proved in [3]. However, for the sake of completeness, we sketch its proof. Let $K$ be a $p$-compact subset of $X$ of the type $K=\left\{\sum_{n \geq 1} \alpha_{n} x_{n}: \bar{\alpha}=\right.$ $\left.\left\{\alpha_{n}\right\} \in B_{l_{q}}\right\}$, where $\bar{x}=\left\{x_{n}\right\} \in l_{p}^{s}(X)$ and $1 / p+1 / q=1$. We can find that $\bar{\beta}=\left\{\beta_{n}\right\} \in c_{0}$ such that $\left\{\beta_{n}^{-1} x_{n}\right\} \in l_{p}^{s}(X)$. Now let us consider the diagonal map $D: l_{q} \rightarrow l_{q}$ defined as $D\left(\left\{\alpha_{n}\right\}\right)=\left\{\alpha_{n} \beta_{n}\right\}$ and $\psi: l_{q} \rightarrow X$ such that $\psi\left(\left\{\alpha_{n}\right\}\right)=$ $\sum_{n \geq 1} \alpha_{n} \beta_{n}^{-1} x_{n}$. Clearly, $D$ is a compact operator, and $\psi$ is a $p$ compact operator. Write $Y=l_{q} / \operatorname{ker} \psi$ and $Q$ for the quotient map from $l_{q}$ to $Y$. Define a $p$-compact operator $\widehat{\psi}: Y \rightarrow X$ as $\widehat{\psi}(Q(\bar{\alpha}))=\psi(\bar{\alpha})$. Now, $Q \circ D\left(B_{l_{q}}\right)$ is a compact set in $Y$. By hypothesis, for this compact set and any $\varepsilon>0$, there exists $R_{0} \in L_{s}(X)$ such that

$$
\sup _{x \in K}\left\|R_{0} x-x\right\|=\sup _{\overline{\bar{\alpha}} \in Q^{\circ} D\left(B_{l_{q}}\right)}\left\|R_{0} \widehat{\psi}(\hat{\bar{\alpha}})-\widehat{\psi}(\hat{\bar{\alpha}})\right\|<\varepsilon .
$$

As $K$ is an arbitrary $p$-compact set, the Banach space $X$ has $L_{s}$ - $p$-AP.

To prove the next result we make use of the following.

Lemma 16. The Banach space $X$ has $L_{s}-p-A P$ for $1<p<\infty$ if and only if

$$
\inf _{R \in L_{s}(X)}\left|\sum_{j \geq 1} \sum_{n \geq 1} \alpha_{n}^{j} f_{j}\left(R x_{n}-x_{n}\right)\right|=0,
$$

where $\bar{x}=\left\{x_{n}\right\} \in l_{p}^{s}(X) ; \bar{\alpha}^{j}=\left\{\alpha_{n}^{j}\right\}_{n \geq 1}$ are in $l_{q}$ for each $j \in \mathbb{N}$ and $\left\{f_{j}\right\}$ in $X^{*}$ satisfying the condition $\sum_{j \geq 1}\left\|\bar{\alpha}^{j}\right\|_{q}\left\|f_{j}\right\|_{X^{*}}<\infty$.

Proof. $X$ has $L_{s}$-p-AP $\Leftrightarrow I \in{\overline{L_{s}(X)}}^{\tau_{p}} \Leftrightarrow \inf _{R \in L_{s}(X)} \mid \varphi(I-$ $R) \mid=0$, for $\varphi$ in $\left(\mathscr{L}(X), \tau_{p}\right)^{*}$. Now the result is immediate from Theorem 2.

Making use of Proposition 4(i) and the above lemma, we further characterize $L_{s}-p$-AP in the following.

Theorem 17. For $1<p, q<\infty$, and $1 / p+1 / q=1$, the following conditions are equivalent.

(i) $X$ has $L_{s}-p-A P$.

(ii) For any Banach space $Y$ and for any $T \in \mathrm{QN}_{p}^{w^{*}}\left(X^{*}, Y\right)$, $T \in \overline{\left\{T R^{*}: R \in L_{s}(X)\right\}}\|\cdot\|$.

(iii) For any reflexive separable Banach space $Y$ and any $T \in Q N_{p}^{w^{*}}\left(X^{*}, Y\right)$, there exists a net $\left\{R_{i}\right\}_{i \in I}$ from $L_{s}(X)$ such that $T R_{i}^{*} \rightarrow T$ in weak operator topology on $\mathscr{L}\left(X^{*}, Y\right)$; that is, $T R_{i}^{*} f \rightarrow \mathrm{Tf}$ in weak topology $\sigma\left(Y, Y^{*}\right)$ of $Y$ for each $f \in X^{*}$.

Proof. (i) $\Rightarrow$ (ii) Let $T \in Q N_{p}^{w^{*}}\left(X^{*}, Y\right)$. As $T$ is $\sigma\left(X^{*}, X\right)-$ $\sigma\left(\mathrm{Y}, Y^{*}\right)$ continuous, range $\left(T^{*}\right) \subset X$ and so $T^{*} \in K_{p}\left(Y^{*}, X\right)$. Now for given $\varepsilon>0$, there exists $R_{0} \in L_{s}(X)$ such that $\left\|R_{0} T^{*}-T^{*}\right\|<\varepsilon$ by Theorem 15. Hence $\left\|T R_{0}^{*}-T\right\|<\varepsilon$, and (ii) follows.

(ii) $\Rightarrow$ (iii) Immediate.

(iii) $\Rightarrow$ (i) For proving that $X$ has $L_{s}-p$-AP, we make use of Lemma 16. Hence we may assume without loss of generality that $\bar{x}=\left\{x_{n}\right\} \in l_{p}^{s}(X), \bar{\alpha}^{j}=\left\{\alpha_{n}^{j}\right\}_{n \geq 1} \in B_{l_{q}}$, for each $j \geq 1$, and $\left\{f_{j}\right\} \subset X^{*}$ satisfies $\sum_{j \geq 1}\left\|f_{j}\right\|_{X^{*}}<\infty$. Consider the map $\psi: l_{q} \rightarrow X$ defined as $\psi\left(\left\{\alpha_{n}\right\}\right)=\sum_{n \geq 1} \alpha_{n} x_{n}$. Then $\psi$ is a $p$-compact map. Hence $\psi^{*}: X^{*} \rightarrow l_{p}$ is a quasi-p-nuclear map by Proposition 4(i); that is, $\psi^{*} \in Q N_{p}\left(X^{*}, l_{p}\right)$. As $l_{p}$ is a reflexive separable Banach space and $\psi^{* *}: l_{q} \rightarrow X^{* *}$, we get range $\left(\psi^{* *}\right) \subset \mathrm{X}$. Consequently, $\psi^{*} \in Q N_{p}^{w^{*}}\left(X^{*}, l_{p}\right)$. Now by hypothesis, there exists a net $\left\{R_{i}\right\}_{i \in I}$ from $L_{s}(X)$ such that $\psi^{*} R_{i}^{*} \rightarrow \psi^{*}$ in weak operator topology on $\mathscr{L}\left(X^{*}, l_{p}\right)$. Hence by uniform boundedness principle, there exists $M \geq 0$ such that $\left\|\psi^{*} R_{i}^{*}\right\| \leq M$, for all $i \in I$. Also we get that $\psi^{*} R_{i}^{*}\left(f_{j}\right) \rightarrow$ $\psi^{*}\left(f_{j}\right)$, for each $j \geq 1$. Choose $R_{i_{0}}$ in $\left\{R_{i}\right\}_{i \in I}$ such that

$$
\left|\left(\psi^{*} R_{i_{0}}^{*}\left(f_{j}\right)-\psi^{*}\left(f_{j}\right)\right)\left(\bar{\alpha}^{j}\right)\right|<\frac{\varepsilon}{2 n_{0}}, \quad \text { for } j=1,2, \ldots, n_{0},
$$


where $n_{0}$ is the integer satisfying

$$
\sum_{j \geq n_{0}+1}\left\|f_{j}\right\|<\frac{\varepsilon}{2\left(M+\|\bar{x}\|_{p}^{s}\right)} .
$$

Now consider

$$
\begin{aligned}
\left|\sum_{j \geq 1} \sum_{n \geq 1} \alpha_{n}^{j} f_{j}\left(R_{i_{0}} x_{n}-x_{n}\right)\right| \\
=\left|\sum_{j \geq 1}\left(\psi^{*} R_{i_{0}}^{*} f_{j}-\psi^{*} f_{j}\right)\left(\bar{\alpha}^{j}\right)\right| \\
\leq \sum_{j=1}^{n_{0}}\left|\left(\psi^{*} R_{i_{0}}^{*} f_{j}-\psi^{*} f_{j}\right)\left(\bar{\alpha}^{j}\right)\right| \\
\quad+\sum_{j \geq n_{0}+1}\left|\left(\psi^{*} R_{i_{0}}^{*} f_{j}-\psi^{*} f_{j}\right)\left(\bar{\alpha}^{j}\right)\right| \\
<\frac{\varepsilon}{2}+\frac{\varepsilon}{2},
\end{aligned}
$$

since $\left(\left\|\psi^{*} R_{i_{0}}^{*}\right\|+\left\|\psi^{*}\right\|\right) \leq M+\|\bar{x}\|_{\mathrm{p}}^{s}$ and $\left\|\bar{\alpha}^{j}\right\|_{q} \leq 1$, for all $j \geq 1$.

Hence $\inf _{R \in L_{s}(X)}\left|\sum_{j \geq 1} \sum_{n \geq 1} \alpha_{n}^{j} f_{j}\left(R x_{n}-x_{n}\right)\right|=0$. Therefore $X$ has $L_{s}$ - $p$-AP by Lemma 16 .

A characterization of $L_{s}^{*}-p$-AP of a Banach space $X^{*}$ is contained in the following.

Theorem 18. $X^{*}$ has $L_{s}^{*}-p-A P$ if and only if, for every Banach space $Y$ and every $T \in Q N_{p}(X, Y), T \in{\overline{\left\{T R: R \in L_{s}(X)\right\}}}^{\|\cdot\|}$.

Proof. The proof of necessity part is omitted as it is analogous to that of (i) $\Rightarrow$ (ii), Theorem 7 .

For converse, let us consider that $T \in K_{p}\left(Y, X^{*}\right)$, where $Y$ is an arbitrary Banach space. Then $T^{*} \in Q N_{p}\left(X^{* *}, Y^{*}\right)$ by Proposition 4(i). Using Proposition 4(ii), we get $T^{*} \epsilon$ $Q N_{p}\left(X, Y^{*}\right)$. Now, by hypothesis, for given $\varepsilon>0$, we can find that $R_{0} \in L_{s}(X)$ such that $\left\|T^{*} R_{0}-T^{*}\right\|<\varepsilon$. Hence $\left\|R_{0}^{*} T-T\right\|<\varepsilon$. Now apply Theorem 15 to get that $X^{*}$ has $L_{s}^{*}-p$-AP.

Finally, we consider a result involving operator ideals and $p$-compact operators, which is analogous to the one proved in [5] for compact operators.

Proposition 19. Let $\mathscr{A}$ be an operator ideal and $Y$ an arbitrary Banach space. If, for $R \in L_{s}(X)$ and any $T \in A^{d}(Y, X), R T$ is a $p$-compact operator from $Y$ to $X$, then for $R \in L_{s}(X)$ and any $T \in \mathscr{A}^{w^{*}}\left(X^{*}, Y\right)$ the operator $R T^{*}$ is also p-compact operator from $Y^{*}$ to $X$. Converse follows when $\mathscr{A} \subseteq Q N_{p}$.

Proof. If $R \in L_{s}(X)$ and $T \in \mathscr{A}^{w^{*}}\left(X^{*}, Y\right)$, then range $\left(\mathrm{T}^{*}\right) \subset$ $\mathrm{X}$. Then $T^{*}=J_{X} T_{a s}$ where $T_{a s}: Y^{*} \rightarrow X$ is the astriction map of $T^{*}$, and $J_{X}$ is the canonical embedding from $X$ to $X^{* *}$. Then $T_{a s} \in \mathscr{A}^{d}\left(Y^{*}, X\right)$ as $T_{a s}^{*}=J_{Y} T \in \mathscr{A}\left(X^{*}, Y^{* *}\right)$. By hypothesis, $R T^{*}=R T_{a s} \in K_{p}\left(Y^{*}, X\right)$.
For converse, consider that $R \in L_{s}(X)$ and $T \in \mathscr{A}^{d}(Y, X)$. Then $T^{*} \in Q N_{p}\left(X^{*}, Y^{*}\right)$ by hypothesis. By Proposition 4(i) $T \in K_{p}(Y, X) \subset K(Y, X) \subset W(Y, X)$. Consequently, range $\left(\mathrm{T}^{* *}\right) \subset \mathrm{X}$ (cf. [14], page 185) (see also [15], page 341). Hence $T^{*} \in \mathscr{A}^{w^{*}}\left(X^{*}, Y^{*}\right)$. Consequently, $R T^{* *} \in$ $K_{p}\left(Y^{* *}, X\right)$ by hypothesis. As $R T=\left.R T^{* *}\right|_{Y}$, the result follows.

\section{References}

[1] A. Grothendieck, "Produits tensoriels topologiques et espaces nucléaires," Memoirs of the American Mathematical Society, vol. 16, no. 16, p. 140, 1955.

[2] P. G. Casazza, "Approximation properties," in Handbook of the Geometry of Banach Spaces, vol. I, pp. 271-316, North-Holland, Amsterdam, The Netherlands, 2001.

[3] J. M. Delgado, E. Oja, C. Piñeiro, and E. Serrano, “The papproximation property in terms of density of finite rank operators," Journal of Mathematical Analysis and Applications, vol. 354, no. 1, pp. 159-164, 2009.

[4] J. Lindenstrauss and L. Tzafriri, Classical Banach Spaces. I: Sequence Spaces, Springer, Berlin, Germany, 1977.

[5] A. Lissitsin, K. Mikkor, and E. Oja, "Approximation properties defined by spaces of operators and approximability in operator topologies," Illinois Journal of Mathematics, vol. 52, no. 2, pp. 563-582, 2008.

[6] M. S. Ramanujan, "Generalised nuclear maps in normed linear spaces," Journal für die Reine und Angewandte Mathematik, vol. 244, pp. 190-197, 1970.

[7] P. K. Kamthan and M. Gupta, Sequence Spaces and Series, vol. 65 of Lecture Notes in Pure and Applied Mathematics, Marcel Dekker, New York, NY, USA, 1981.

[8] M. Gupta and A. Bhar, "on $\lambda$-compact operators," Indian Journal of Pure and Applied Mathematics, vol. 44, no. 3, pp. 355-374, 2013.

[9] D. P. Sinha and A. K. Karn, "Compact operators whose adjoints factor through subspaces of $l_{p}$," Studia Mathematica, vol. 150, no. 1, pp. 17-33, 2002.

[10] Y. S. Choi and J. M. Kim, "The dual space of $\left(L(X, Y), \tau_{p}\right)$ and the $p$-approximation property," Journal of Functional Analysis, vol. 259, no. 9, pp. 2437-2454, 2010.

[11] J. M. Delgado, C. Piñeiro, and E. Serrano, "Operators whose adjoints are quasi p-nuclear," Studia Mathematica, vol. 197, no. 3, pp. 291-304, 2010.

[12] A. Pietsch, Operator Ideals, vol. 16 of Mathematische Monographien, VEB Deutscher Verlag der Wissenschaften, Berlin, Germany, 1978.

[13] M. Gupta and A. Bhar, "Generalized Orlicz-Lorentz sequence spaces and corresponding operators ideals," Mathematica Slovaca, vol. 64, no. 6, 2014.

[14] J. B. Conway, A Course in Functional Analysis, vol. 96 of Graduate Texts in Mathematics, Springer, New York, NY, USA, 1985.

[15] R. E. Megginson, An Introduction to Banach Space Theory, vol. 183 of Graduate Texts in Mathematics, Springer, New York, NY, USA, 1998. 


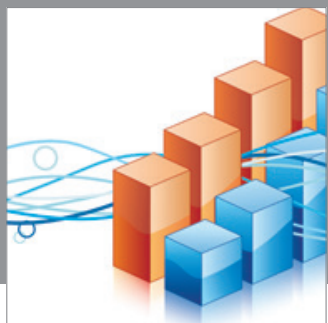

Advances in

Operations Research

mansans

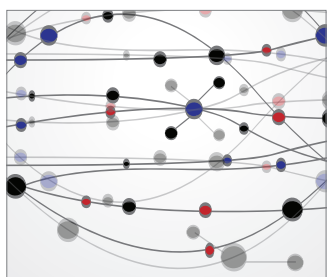

The Scientific World Journal
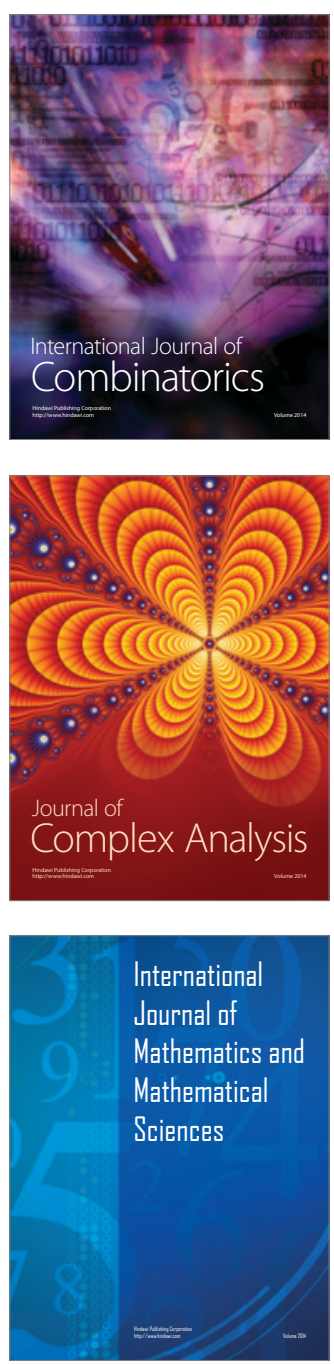
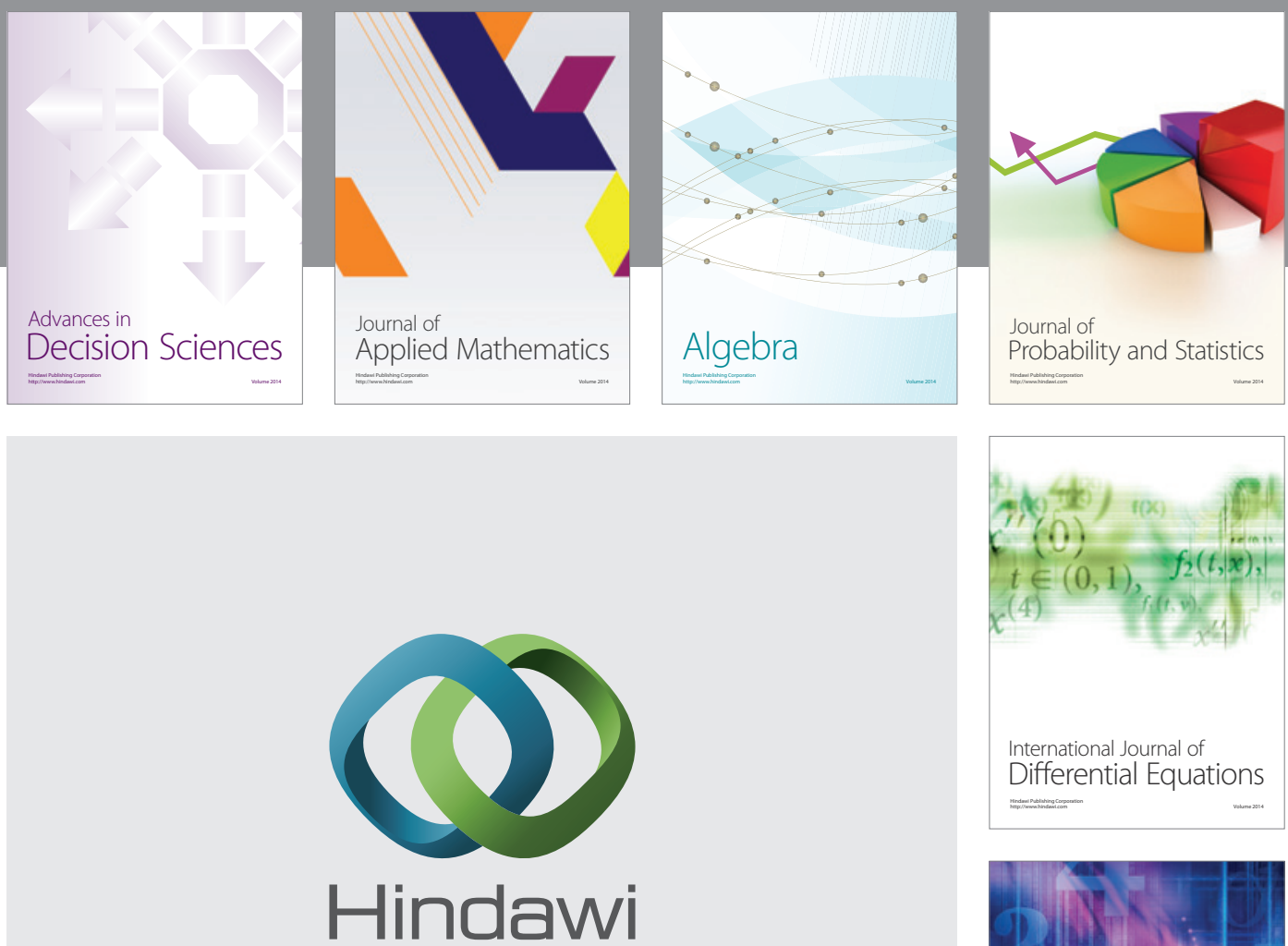

Submit your manuscripts at http://www.hindawi.com
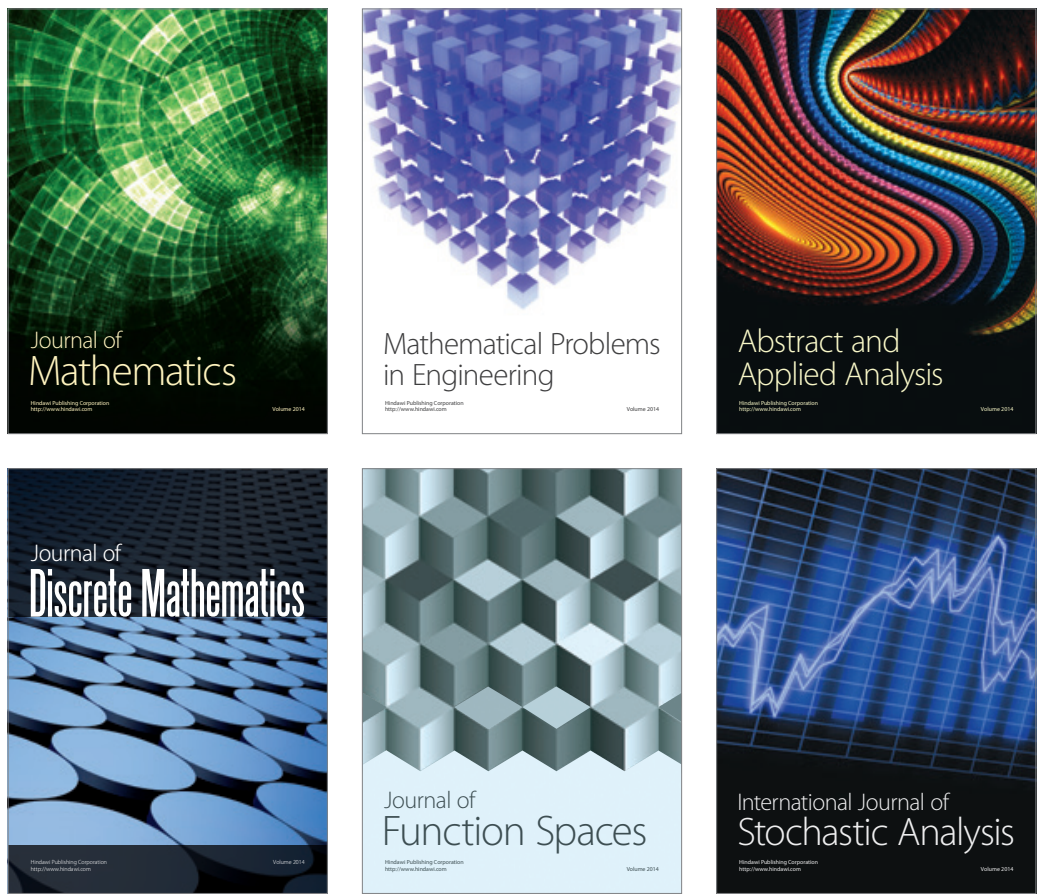

Journal of

Function Spaces

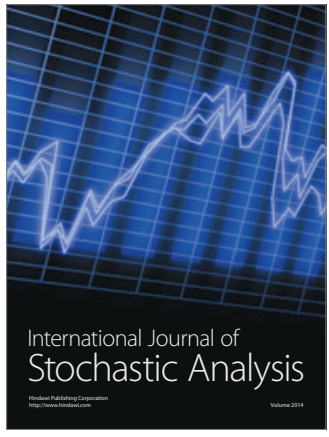

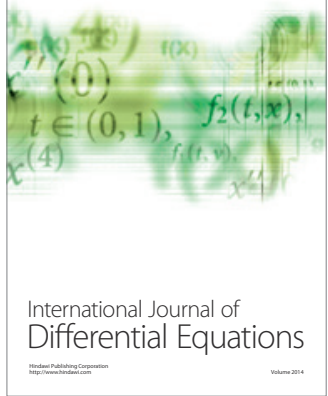
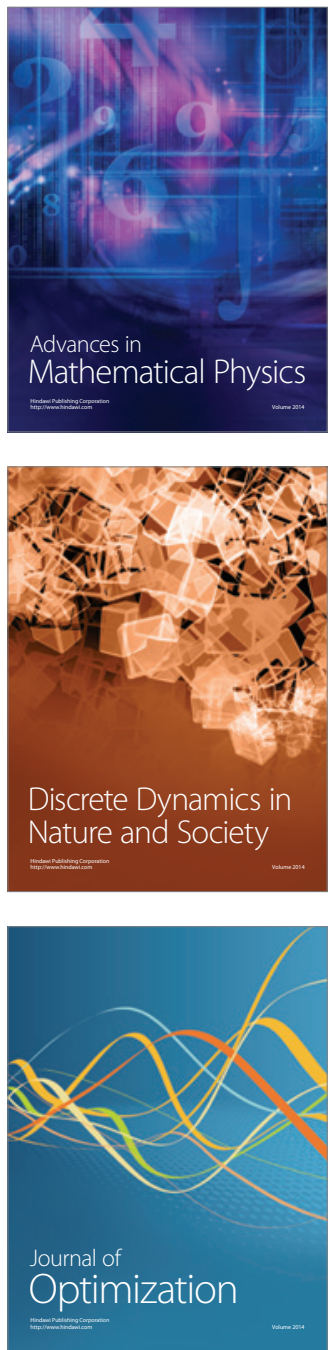